\title{
Automatic Measurement of Blood Vessel Angles in Immunohistochemical images of Liver Cancer
}

\author{
Hongbin Zhang ${ }^{1, a \#}$,Kun Zhang ${ }^{1, b \#}$,Li Chen ${ }^{2, c}$,Jianguo Wu ${ }^{1, d}$, Peijian Zhang ${ }^{1, e}$, \\ Huiyu Zhou ${ }^{3, f}$ \\ ${ }^{1}$ School of Electrical Engineering, Nantong University, Nantong, China \\ ${ }^{2}$ Department of Pathology, Medical College, Nantong University, Nantong, China \\ ${ }^{3}$ School of Electronics, Electrical Engineering and Computer Science \\ Queen's University Belfast \\ a1951299040@qq.com, ${ }^{b}$ zhangkun_nt@163.com, ${ }^{c} b 11 @$ ntu.edu.cn, ${ }^{\mathrm{d}}$ wu.jg@ntu.edu.cn \\ (corresponding author), ${ }^{\mathrm{e}} \mathrm{zh}$ ang.pj@ntu.edu.cn, ${ }^{\mathrm{f}}$.zhou@ecit.qub.ac.uk
}

\begin{abstract}
Measurement of vascular angle is a key step in quantitative analysis of immunohistochemistry. This paper presents a method for automated measurement of vascular angle in immunohistochemical images of liver cancer. Firstly, Colour Deconvolution is used to conduct stain separation on a H\&Estained immunohistochemical image, and then blood vessels are segmented using an improved Otsu algorithm. Then the standard SURF algorithm is used to select feature points of the image, and then these feature points are divided into two equal groups according to the distance between individual feature points and the far left (or right) feature point. Finally, a standard least squares method is used to fit two lines using the two group of points. When the linear deviation of the fitting result based on the two groups of feature points is significant, it is necessary to adjust the belonging of the points of the two groups, and then the two sets are fitted again respectively till the correlation coefficients of the two fitted lines are greater than the predefined threshold, meaning that the measurement of the blood vessel angle in the immunohistochemical map is completed. In the experiments, 45 liver cancer images are used, where about 600 vessels are extracted. Compared with the experts' results, our proposed technique results in better accuracy. It is worthy to point out that, to our knowledge, our system is the first one that conducts automated measurement of blood vessel angle of immunohistochemistry.
\end{abstract}

Keywords: immunohistochemical image; color deconvolution; image segmentation; feature extraction; least square

\footnotetext{
\# These authors contributed equally to this work and should be considered co-first authors
} 


\section{Introduction}

Modern medicine shows the occurrence of liver cancer is a multi-factor, multi-step biological process. The proliferation and metastasis of liver cancer cells relies on the growth of new blood vessels. The development of blood vessels and liver cancer's growth, infiltration and recurrence are closely correlated. Clinically, when liver cancer progresses, vascular segments may become irregular and displaced, and liver capillaries can be deformed (e.g. hook-shaped). Therefore, we need to develop a method to identify the deformed blood vessels and then calculate the vascular angle. The stage immunohistochemical(IHC) technique [1] has been used to improve the accuracy of the clinical pathological diagnosis, and become an important evaluation standard for differentiating benign and malignant tumors. However, the current automated IHC analysis method is not enough to solve all the morphological problems of blood vessels, when only statistical information such as a blood vessel's length, area, aspect ratio and so on. At present, there is no system that can reliably calculate vascular angles, which is affected by individual subjective factors only. Therefore, the automated angle measurement algorithm proposed in this paper is of great significance as it helps understand the growth and metastasis of malignant tumors.

Immunohistochemistry is the core technology in biological image analysis, and the improvement of biomedical image processing technology has promoted the development of the automated analysis tools of IHC images, which is of great significance for a pathologist to identify disease related biological activities. In case staining agents are uneven or image noise is severe, accurate extraction of vascular structures for auxiliary diagnosis becomes challenging, which will significantly affect the accuracy of subsequent quantitative analysis.

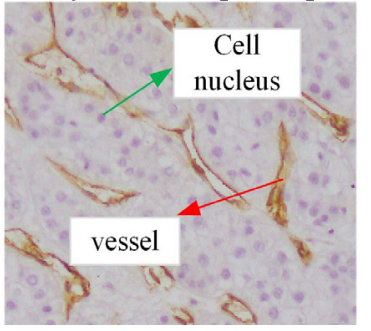

(a)

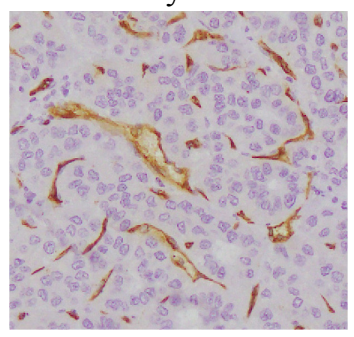

(b)

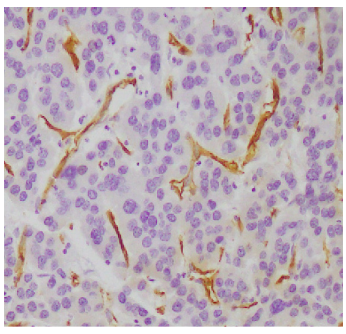

(c)

Fig. 1: IHC images of liver cancer (magnification of $400 \times$ ) respectively for the wellsegmented, moderately segmented and poorly segmented stage.

Because IHC images accompany a variety of colorants, traditional methods cannot be used directly to achieve the purpose of accurate segmentation. For example, Ref. [2] reports a method of using $R \backslash G \backslash B$ components and color difference as a basis for the division. However, due to the strong correlation in the three color space $R \backslash G \backslash B$, it is difficult to achieve color separation for immunohistochemical positive, negative and background using the RGB color model. Some scholars have transformed the RGB color model into a HSI model [3] or a CMYK model [4] for multi-color image segmentation, but these methods rely on the thresholding setting, which can easily 
lead to the loss of a large amount of information. In order to solve the problem of separation of various colors in the IHC image, Ruifrok proposed a method of color deconvolution [6] to separate different colored tissue components and form multiple single-color images. Recent research reports show that, on the basis of color separation, an appropriate segmentation algorithm can be used to achieve the purpose of separating various structures accurately [6].

Blood vessels have tree-like shapes, and some of them look like slender lines or tubes. The shape of a blood vessel determines the characteristics of image segmentation. There are many ways to divide blood vessels, but to our knowledge no method has been available to handle different environments. In [7], a new approach was proposed to detect the edge of a blood vessel by analyzing the pixels perpendicular to the tracked image point. The disadvantage of this algorithm is that people need to assign a starting and an end points. The maximum variance algorithm proposed in [8] has a good effect on the segmentation of images. The basic idea is to rendering the best threshold for the staining separation, which is not affected by the brightness of the images and the gray levels.

In the analysis, feature extraction and matching of images is very important. Commonly used feature extraction and matching algorithms include SURF [9] and SIFT [10]. The main advantage of the SURF algorithm is to keep the robustness of SIFT and to reduce feature detection time greatly. This algorithm is actually an acceleration version of the standard SIFT algorithm, but also has reduced computational time.

The image registration method proposed in this paper is using image features. In general, this paper introduces a method for automated identification of blood vessels. This method, based on the selection of the characteristic points of images, performs line fitting and then derives the angle between lines to represent the deflection of a blood vessel.

\section{Method}

According to the characteristics of IHC images, our proposed algorithm includes the following steps: Firstly, a multi-colored IHC image passes through the Color Deconvolution step [6], then the corresponding single colored image is obtained. An improved OTSU algorithm [8] is used to obtain coarse image segmentation of potential vessels, then the smoothed $\mathrm{t}$ boundaries and holes is solved through morphological processing. The standard SURF algorithm is used to extract feature points of the potential blood vessels, and a least square method [11] is used for fitting points of clouds to determine the position of two straight lines. Finally, using the common geometric relationship, we can calculate the vascular angle. 


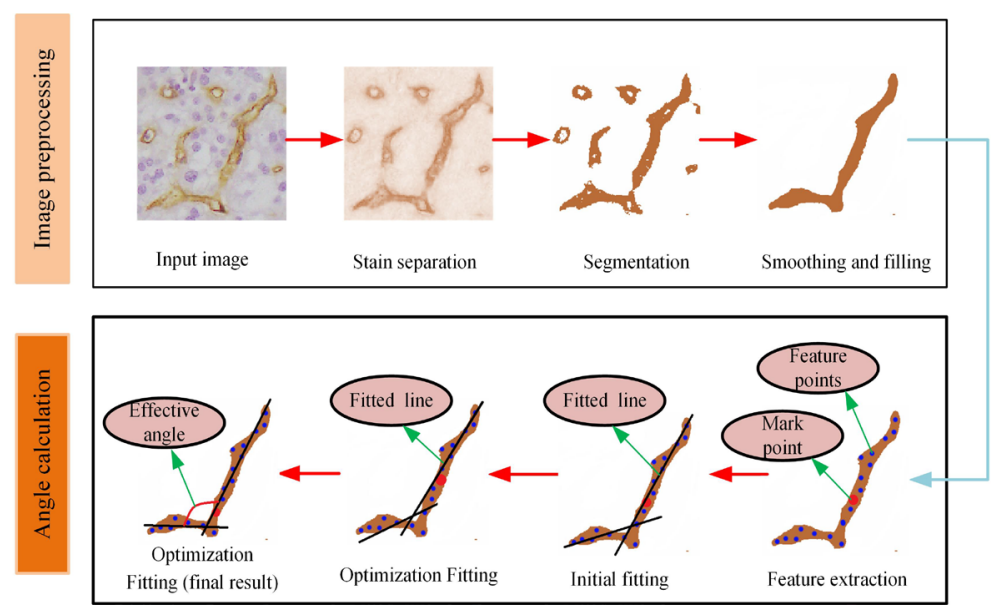

Fig. 2. Flowchart of image preprocessing and angle calculation

\subsection{Linear fitting}

In practice, it is not trivial to calculate the angle between two fitted lines from the extracted feature points of blood vessels due to the clutters and image noise. To solve this problem, we use the following method:

We extract the coordinate information of feature points of blood vessel, and number all the $\mathrm{N}$ feature points:

(1) According to the coordinate positions of the feature points, we randomly choose the first endpoint, denoted as $P_{1}$;

(2) We calculate the distance between the endpoint $P_{1}$ and the other $N-1$ feature points. The $2^{\text {nd }}$ endpoint $P_{2}$ is the one that has the shortest distance to the first endpoint. We then calculate the distance between $P_{2}$ and the other $N-2$ points respectively. When $\left|P_{i} P_{2}\right|+\left|P_{i} P_{1}\right|$ has the minimum value, $i$ denoted as $P_{3}$. We perform such an iteration till all the feature points are marked.

When $N$ is an odd number, the feature point with the number $(N+1) / 2$ is the marked point. However, when $N$ is an even number, $\mathrm{N} / 2$ or $(\mathrm{N} / 2)+1$ is taken as the marker. The $\mathrm{N}$ feature points of the blood vessel are divided into two equal sets $S_{1}$ and $S_{2}$ (when $\mathrm{N}$ is an even number, $S_{1}=\left\{P_{1}, P_{2}, \ldots, P_{N} / 2\right\}, S_{2}=\left\{P_{N} / 2+1, \ldots\right.$, $\left.P_{N}\right\}$. When $N$ is an odd number, $\left.S_{1}=\left\{P_{1}, \ldots,\left(P_{N}-1\right) / 2\right\}, S_{2}=\left\{\left(P_{N}+1\right) / 2, \ldots, P_{N}\right\}\right)$. The least square fitting is performed to obtain two straight lines $L_{1}$ and $L_{2}$. More details follow:

(1) When fitting the two straight lines $L_{1}$ and $L_{2}$, if the correlation coefficient (an indicator of the good fitting) is greater than 0.8 , we determine the two straight lines as the outputs;

(2) Otherwise, denoted as $L_{x}$, the marked points moves away a position randomly, leading to $S_{1}=\left\{P_{1}, P_{2}, \ldots, P_{N} / 2, P_{N} / 2+1\right\}, S_{2}=\left\{P_{N} / 2+1, \ldots, P_{N}\right\}$ We perform 
line fitting again. If the correlation coefficient $\rho$ becomes smaller, the marked point moves to the opposite direction, leading to $S_{1}=\left\{P_{1}, P_{2}, \ldots, P_{N} / 2-1\right\}, S_{2}=$ $\left\{P_{N} / 2, P_{N} / 2+1, \ldots, P_{N}\right\}$.

(3) Once the two straight line positions have been determined, we calculate the angle formed by the intersection of the two straight lines, which is the final blood vessel angle.

\section{Linear fitting method simulation}

Input: All the $N$ characteristic points in a vessel

Output: The angle of the blood vessel

1. divide these feature points into two equal sets $S_{1}$ and $S_{2}$

2. $S_{1}, S_{2}$ least squares straight line fitting, denoted as $L_{1}, L_{2}$

3. calculate the correlation coefficients of lines $L_{1}$ and $L_{2}$, denoted as $\rho_{1}, \rho_{2}$

4. if $\rho_{1}>0.8$ and $\rho_{2}>0.8$, then

5. calculate the angle between lines $L_{1}$ and $L_{2}$ end

6. else if

7. mark point move a point to the left (or right), constitute two new set $S_{1}^{\prime}, S_{2}^{\prime}$

8. $S_{1} S_{2}$ least squares straight line fitting, denoted as $L_{1}, L_{2}$

9. calculate the correlation coefficients of lines $L_{1}$ and $L_{2}$, denoted as $\rho_{1}, \rho_{2}$

10. if $\rho_{1}>0.8$ and $\rho_{2}>0.8$, then

11. calculate the angle between lines $L_{1}$ and $L_{2}$ end

12. else if

13. $\left(\rho_{1}+\rho_{2}\right)>\operatorname{initial}\left(\rho_{1}+\rho_{2}\right)$ then

14. the marker moves a point in the same direction, constitute two new set $S_{1}^{\prime}, S_{2}^{\prime}$

15. repeat steps $8-11$

16. else if $\left(\rho_{1}+\rho_{2}\right)<$ initial $\left(\rho_{1}+\rho_{2}\right)$ then

17. the marker moves a point in the opposite direction, constitute two new set $S_{1}^{\prime}, S_{2}^{\prime}$

18. repeat steps $8-11$.

The processing steps of the proposed linear fitting scheme can be found in Fig. 3. 

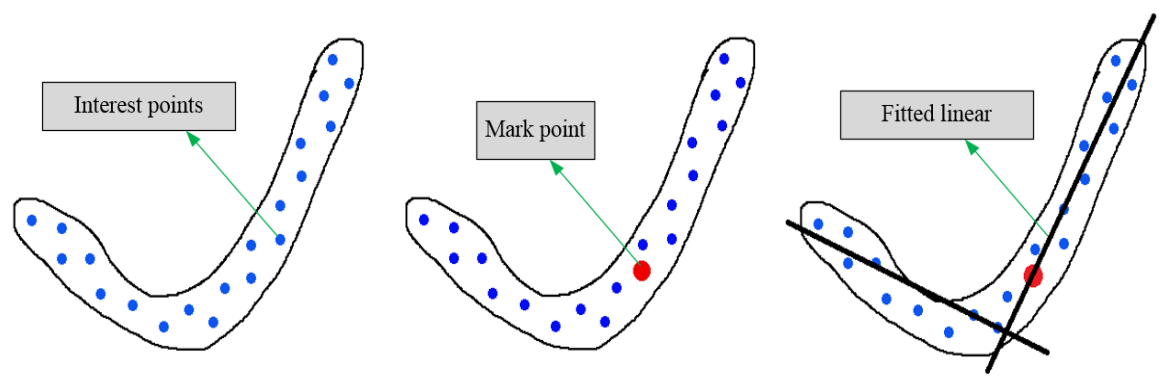

1. Select feature points

2. Divided into two sets

3. Linear fitting

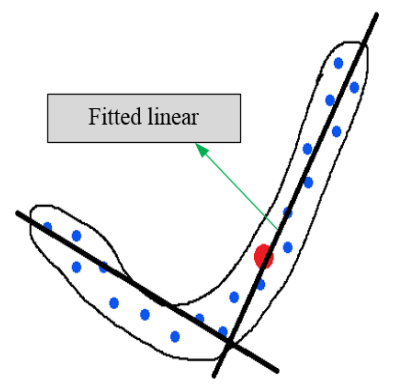

4. Optimum fitting (1)

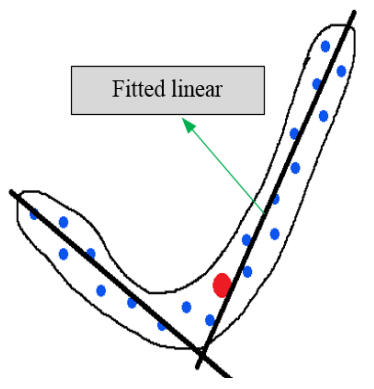

5. Optimum fitting (2)

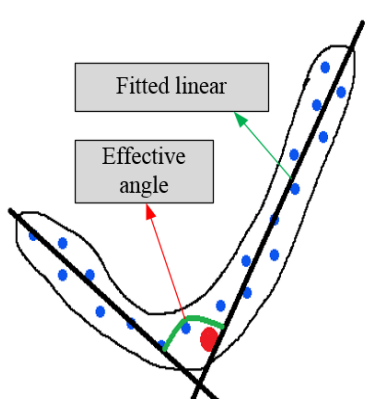

6. Optimum fitting (3)

Fig. 3. Linear fitting process.

\subsection{Proposed Framework}

\section{Step1: Staining separation}

The color deconvolution algorithm [6] is to obtain independent information for each color. In order to get a correct color absorption factor, the orthogonal transformation of the RGB is needed, which can be achieved by the color deconvolution method.

\section{Step 2: Segmentation}

Based on the empirical exercises, mean variance of immunohistochemical images shows much irregular changes. The traditional Otsu algorithm cannot handle this challenge. In this paper, the optimal threshold criterion function of the improved Otsu algorithm is:

$$
T^{*}=\operatorname{Arg} \max _{0 \leqslant T \leqslant L-1}\left(\sigma_{B}^{2}\right)
$$

\section{Step 3: Feature extraction}

The extraction of SURF feature points can determine the location of interest points through calculating the Hessian matrix determinant of the local extreme points. The SURF algorithm [9] uses the box filter instead of the Gaussian, and introduces an integral image, which greatly improves the operation speed and accuracy. In light of the convolution operation of the box filter, the results include determinants Dxx, Dxy and Dyy. Thus, the determinant of the Hessian matrix can be reduced to:

$$
\operatorname{Det}(H)=D_{x x} D_{y y}-\left(w D_{x y}\right)^{2}
$$


Where $\mathrm{W}$ is the weight coefficient, which is appropriately given as 0.9 . In accordance with the approximate calculation method of the determinant of the Hessian matrix, the response in the sigma scale is gained.

\section{Step 4: Linear fitting}

Linear fitting is commonly achieved by a least square method, which aims to work out the linear relationship between the inputs and the parameter sets. When "deviation square sum" between the test data points and the fitting line is the least, the best fitting straight line is obtained.

In statistics, the correlation coefficient is used to measure the degree of correlation between the degree of closeness.

$$
\rho_{X Y}=\frac{\operatorname{Cov}(X, Y)}{\sqrt{D(X)} \sqrt{D(Y)}}
$$

In the above formula, $\operatorname{Cov}(X, Y)$ is the covariance of $X$ and $Y, D(X) D(Y)$ are the variance of $X$ and $Y$, respectively.

When $\left|\rho_{X Y}\right|=1, \mathrm{X}, \mathrm{Y}$ is perfectly correlated;

When $\left|\rho_{X Y}\right|<1$, the larger the absolute value of $\mathrm{P}_{\mathrm{XY}}$, the bigger the changes that $\mathrm{X}$ causes $\mathrm{Y}$ to change;

When $\left|\rho_{X Y}\right|>0.8$, the relationship between $\mathrm{X}$ and $\mathrm{Y}$ is known as highly relevant;

When $\left|\rho_{X Y}\right|<0.3$, the relationship between $\mathrm{X}$ and $\mathrm{Y}$ is called low correlation, and others are moderate correlation.

\section{Step 5: The angle calculation}

$$
\mathrm{a}=\arctan \left|\frac{k_{2}-k_{1}}{1+k_{1} k_{2}}\right|
$$

Where $\mathrm{k}_{1}$ and $\mathrm{k}_{2}$ were two straight lines.

\section{Experiments and Results}

In this paper we study the IHC images of 4 grades of liver cancer, each of which contains 20 cases. The samples come from 82 patients with liver cancer surgery in the Affiliated Hospital of Nantong University from January to September 2016. There were 68 males and 14 females, aged from 31 to 70, with an average age of $(54.7 \pm$ $10.5)$ years. No interventional therapy has been applied before the operation.

All of them had CPA examination within one week before the surgery, and postoperative pathology and immunohistochemistry after the surgery. All the pathological sections were treated with CD34 monoclonal antibody, H\&E staining and IHC staining, whilst IHC shows positive, and we adopted a Olympus DP27 camera, which has the exposure time of 1/260 seconds, the horizontal and vertical resolution is $72 \mathrm{dpi}$, and the image size is $1224 \times 960$ with $400 \times$ magnification.

Clinically, according to the different levels of cell differentiation, malignant tumors are often divided into three groups: high differentiation, differentiation, and undifferentiated. Highly differentiated tumors: the differentiation degree of tumor 
cells is high, and the deflection of blood vessels is not obvious, falling between 135180 degree.

Moderately differentiated tumors: the degree of differentiation of tumor cells is low, and there are some changes to the deflection of blood vessels, falling in 105-135 degree.

Poorly differentiated tumors: the degree of differentiation of cancer cells is lower, and the angle of deflection of blood vessels is obvious, most of which are between 75105 degree.

Each stage of the clinical study is conducted by the single factor analysis of variance. Bivariate correlation analysis is performed in different pathological stages. The $t$ experiment is performed to compare the differences in a group, and the variance analysis is used to compare across groups, where $\mathrm{P}<0.05$ indicating statistical validity. All the data is analyzed using SPSS10 for windows.

\subsection{IHC image preprocessing}

The method designed in this paper is mainly used for IHC vascular angle measurement. For the stained IHC image, it is necessary to separate the brown blood vessels from the background cells. The extraction of blood vessels is performed by using the classical color deconvolution method. Fig. 4 shows the color differentiation one step by one step.

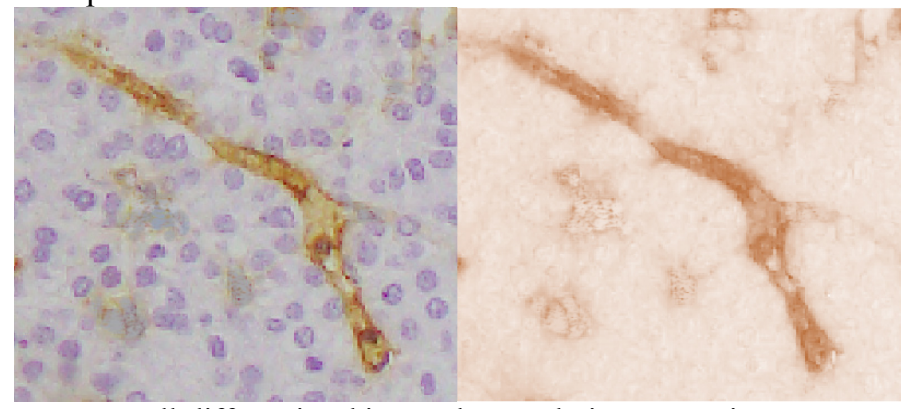

Well-differentiated image deconvolution processing

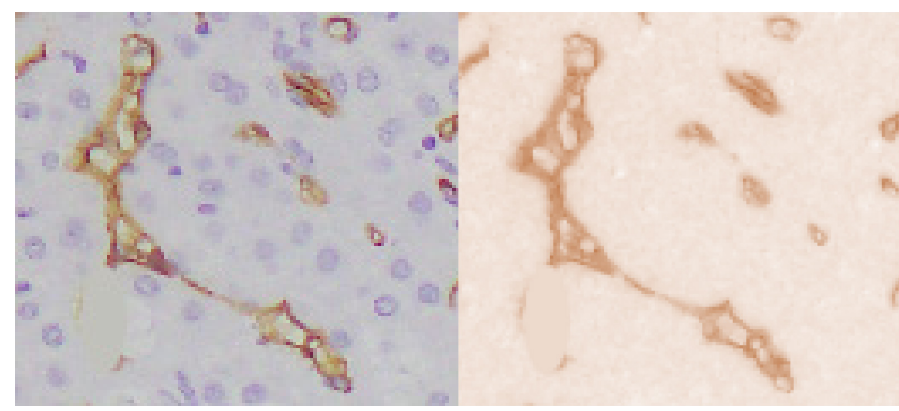

Moderately differentiated image deconvolution processing 


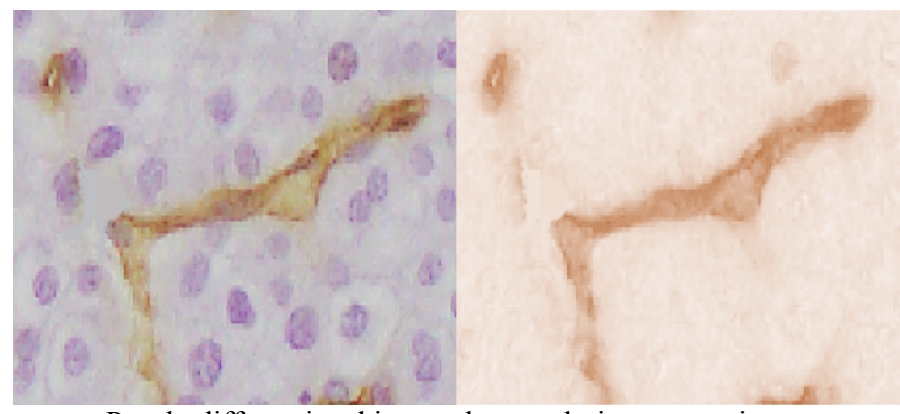

Poorly differentiated image deconvolution processing

Fig. 4. Color deconvolution for color sepration.

\subsection{Improved OTSU algorithm}

The determination of segmentation thresholds is the key factor to determine the accuracy of feature extraction by analyzing the characteristics of the gray histogram of the image and combining with the improved OTSU algorithm mentioned above. Fig. 5 illustrates the procedure of applying the improved OTSU algorithm.

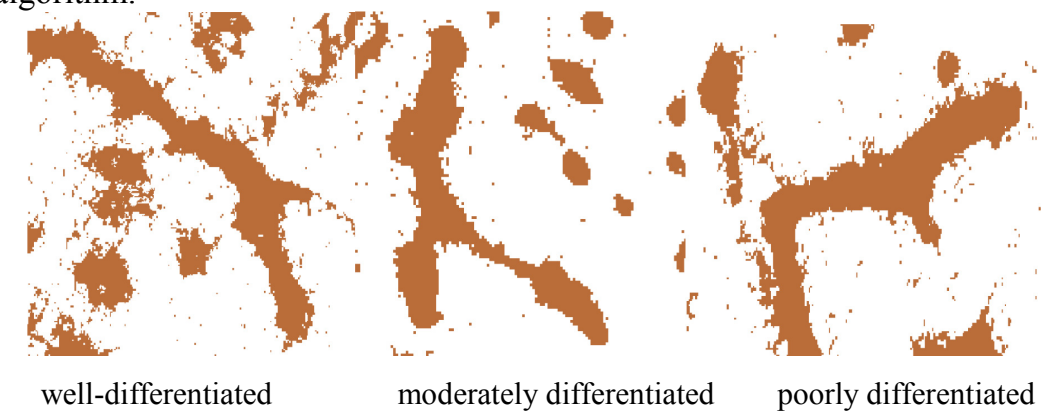

(a)

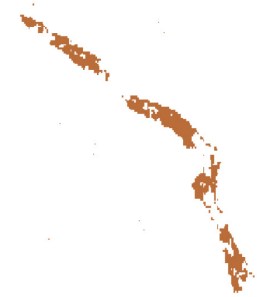

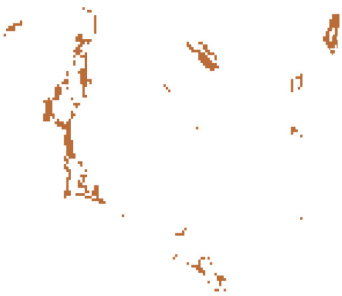

moderately differentiated

(b) 


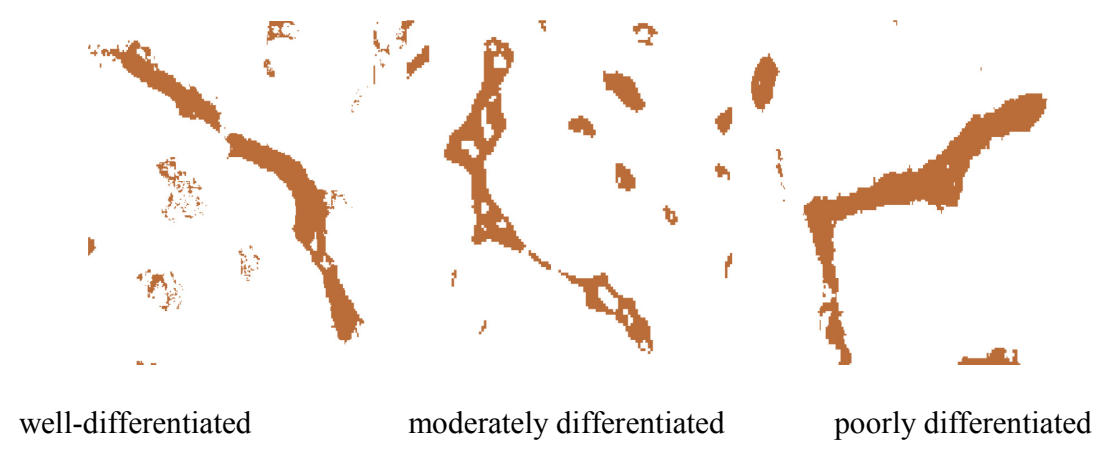

(c)

Fig. 5. Comparison of several typical threshold segmentation methods, among them, (a), (b) and (c) were obtained using percentile [12], shanbhag[13] and Otsu methods[8], respectively

It can be seen from Fig. 5, after the series of image processing, immunohistochemical images only contain the characteristic area of blood vessels (the target feature) and the background area. After a binary operation, we obtain Fig. 6. It can be seen that the immunohistochemical vascular map created by the improved OTSU method has good connectivity and high signal-to-noise ratio, which is an effective in this case.
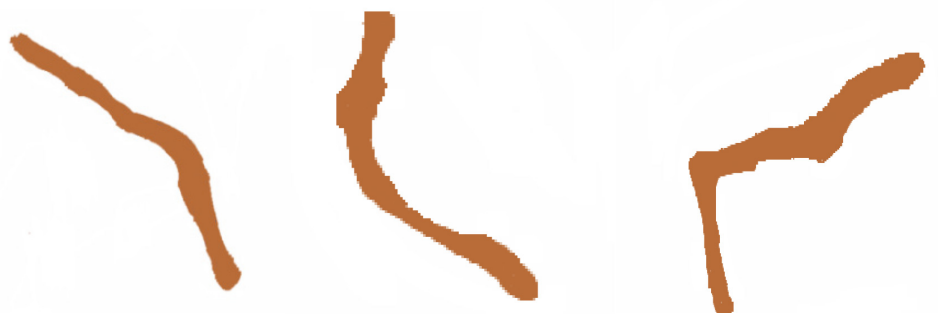

Fig. 6. Illustration of smoothing and filling.

\subsection{Feature extraction}

In order to ensure the usefulness of the feature points and eliminate other interference, it is necessary to denoise the image after the Otsu thresholding process. We use the standard SURF algorithm[9] to deal with the results of the three pathological stages as shown in Fig. 7.
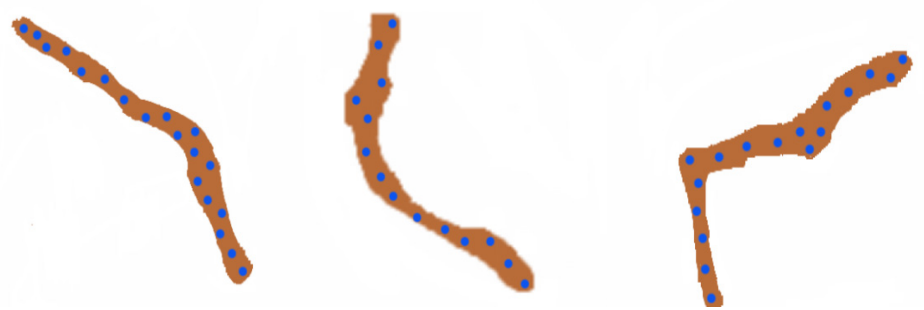

Fig. 7. Illustration of SURF feature extraction. 


\subsection{Least square fitting}

In practical applications, the ration of the length and width of a blood vessel is normally 5 or higher. After having applied the standard least square method, we have the results shown in Fig. 8.
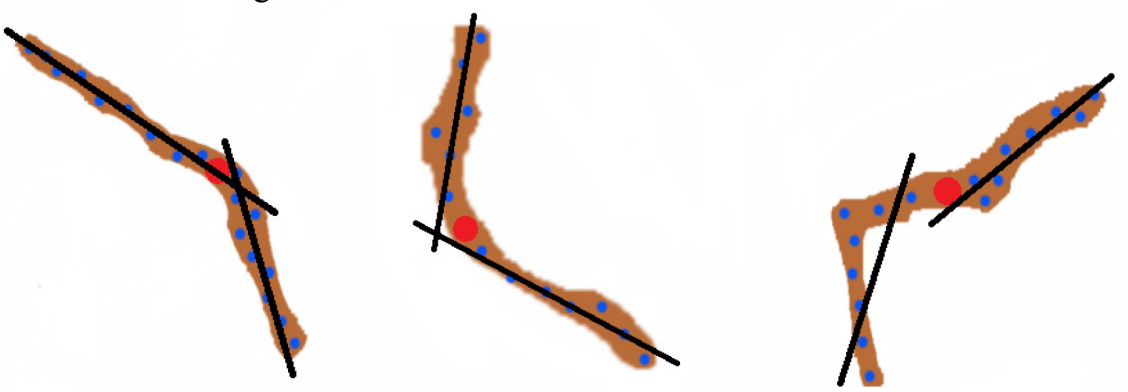

Fig. 8. Preliminary linear fitting results.

As can be seen from Fig. 8, well-differentiated and moderately differentiated blood vessels have been obtained by confident line fitting. For poorly differentiated blood vessels, one of the fitting lines has significant deviation. This requires the moving of the marked point and a proper re-fitting operation accordingly.

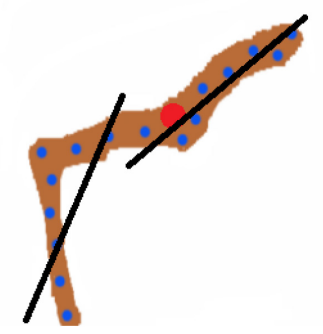

Fig. 9. The marker points are randomly moved by one unit and re-fitted

From Fig. 9, if the marked point is moved to right, the fitting line's deviation increases significantly, therefore we continue to move the marked point to the left. This results in Fig. 10.

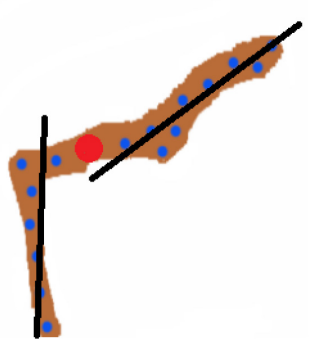

Fig. 10. When the correlation coefficient is reduced, we move the marked point to the opposite direction and then re-fit the line. 
By calculating the linear correlation coefficient, we can see that the deviation of the line fitting is dramatically reduced and the position of the marked point moves. We will obtain Fig. 11.

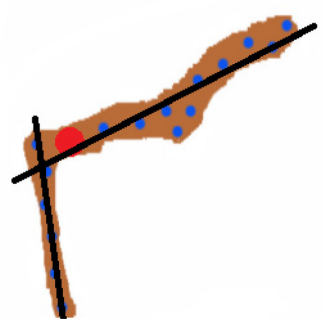

Fig. 11. When the correlation coefficient increases, we move the marked point in this direction and then re-fit the line.

By changing the position of the marked points, we optimize the linear fitting. According to the geometric relationship of the plane, the angle/deflection of a blood vessel can be calculated. However, there are some errors in the fitting of the feature points, and this needs to be investigated in the future work.

According to the geometric relationship within the plane, the angle of the blood vessel can be calculated accurately. However, there are some errors in the fitting of the feature points and the least squares method, and it is not easy to observe in the statistical analysis. Therefore, the method of classifying the blood vessel angle in each image is calculated so as to analyze the result by using the statistical principle.

In the three groups of liver cancer IHC images (about 20 cases were studied), the following statistics are derived:

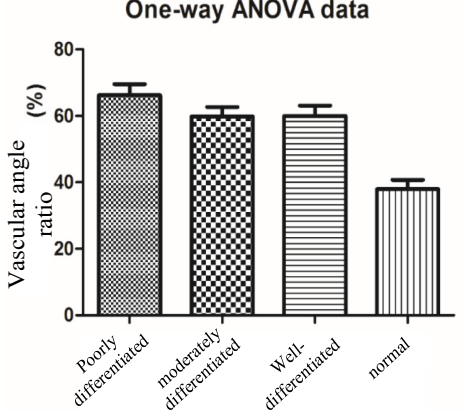

(a)

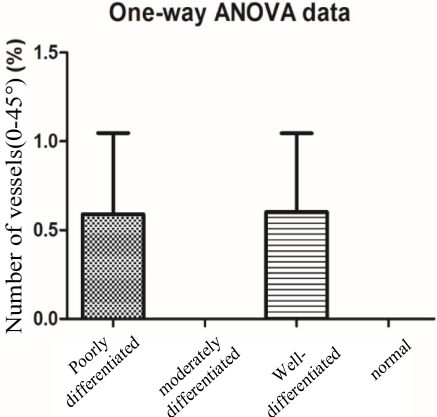

(b) 


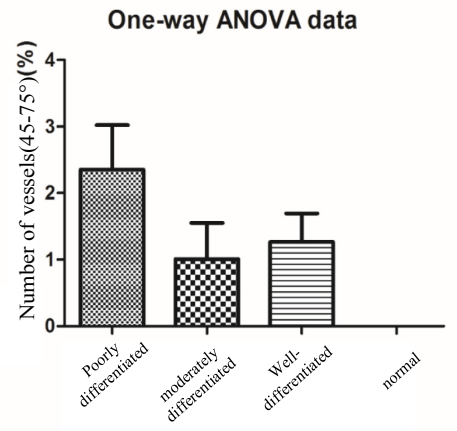

(c)

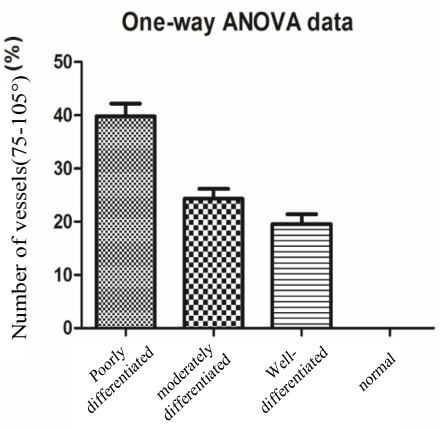

(d)

Fig. 12. The variance analysis of the number of vessels in different stages. The value of $\mathrm{P}$ is a probability, which reflects the probability of occurrence of an event. Statistics based on the significance of the $\mathrm{P}$ value obtained by the significance test, the general $\mathrm{P}<$ 0.05 is significant, $\mathrm{P}<0.01$ is very significant, among them, (a) $\mathrm{p}<0.0001$; (b) $\mathrm{p}=0.0026$; (c) p $<0.0001 ;$ (d) p $<0.0001$.

The explanation of $\mathrm{P}$ value greater than 0.05 is not that the test factors have no effect, but can not yet determine the test factors have a role. The meaning of the $\mathrm{P}$ value is simply that the source of the difference is the possibility of sampling error (random error). Usually the definition of $\mathrm{P}$ is less than 0.05 that have statistical significance, meaning when the possibility of differences can be used to explain the sampling error of less than 5\%, we would think that this has nothing to do with the sampling error, but caused by the factors of the test. The results of Fig. 12 show that the experimental results are statistically significant, and the method proposed in this paper can produce reasonably correct vascular angles.

\section{Conclusion}

This paper introduces a new method of measuring vascular angles as the indicator of different stages of liver cancer. IHC images of liver cancer were processed through Color Deconvolution to separate blood vessels and cells. The feature points of the region of interest were extracted using SURF, fitted by a least square method. Most of the blood vessels shown in the IHC images satisfied the requirements. However, for a small number of complex blood vessels, the use of the current method cannot produce satisfactory results. In the future work, we intend to closely look into the segmentation stage, whilst removing the image background noise. We also compare our proposed method against the other state of the art technologies.

Acknowledgements: This work was financially supported by Nantong Research Program of Application Foundation under Grant No. GY12016022, and Dr. H. Zhou is supported by UK EPSRC under Grant P/N011074/1, and Newton Advanced Fellowship under Grant NA160342. 


\section{References}

1. Bhat, Payal, Singh, Nittin Dev, Leishangthem, Geeta Devi,etc:Histopathological and immunohistochemical approaches for the diagnosis of Pasteurellosis in swine population of Punjab.J. VETERINARY WORLD.9,989--995(2016)

2. Reeves, Anthony P., Liu, Shuang, Xie, Yiting: Image segmentation evaluation for verylarge datasets.In: Conference on Medical Imaging - Computer-Aided Diagnosis,9785. ,San Diego, CA(2016)

3. Abuturab, Muhammad, Rafiq:Multiple color-image authentication system using HSI color space and QR decomposition in gyrator domains. J. JOURNAL OF MODERN OPTICS.63,1035--1050(2016)

4. Jun Qian, Feng Gao, Hui-Hui Liu:The color properties of a reflective CMYK module using polymer-dispersed liquid crystal (PDLC). J.OURNAL OF THE SOCIETY FOR INFORMATION DISPLAY.21,511--516(2013)

5. Onder, Devrim, Zengin, Selen, Sarioglu, Sulen: A Review on Color Normalization and Color Deconvolution Methods in Histopathology. J.APPLIED IMMUNOHISTOCHEMISTRY \& MOLECULAR MORPHOLOGY.22,713--719(2014)

6. Ruifrok, AC.: Comparison of quantification of histochemical staining by Hue-SaturationIntensity ( HSI) transformation and color deconvolution. J. Appl Immunohistochem Mol Morphol.11,85- 91(2004)

7. Calamante, Fernando, Tournier, Jacques-Donald, Kurniawan, Nyoman D.etc: Superresolution track-density imaging studies of mouse brain: Comparison to histology. J. NEUROIMAGE.59,286--296(2012)

8. Zhongshan Chen, Yan Tu:Improved image segmentation algorithm based on OTSU algorithm. J. International Journal of Advancements in Computing Technology.4,206-215(2012)

9. Lei Zhang, Yongsheng Dong, Jiexin Pu:Object recognition based on SURF. J. ICIC Express Letters, Part B: Applications.6,259--264(2015)

10. Mu Yi Yin, Fei Guan, Peng Ding, ZhongFeng Liu:Implementation of image matching algorithm based on SIFT features. J.Applied Mechanics and Materials. 602--605,3181-3184(2014)

11. Bei Xie,Bose, Tamal:Partial update least-square adaptive filtering.Synthesis Lectures on Communications.7,1-115(2014)

12. Upadhyay, Kanchan,Asthana, Anupama, Tiwari, Neetu,Mathew, Snitha B.:Determination of nimesulide in pharmaceutical and biological samples by a spectrophotometric method assisted with the partial least square method.Research on Chemical Intermediates.39,3553-3563(2013)

13. Weiwei Fan, Dongsheng Zhao, Songjun Wang:A fast statistics and analysis solution of medical service big data.Proceedings - 2015 7th International Conference on Information Technology in Medicine and Education.itme2015,9--12(2016)

14. Kapitány, Kristóf, Barsi:Deriving hierarchical statistics by processing high throughput medical images. IFMBE Proceedings.50,32--35(2015)

15. Mercure, Elizabeth,Destrempes, François, Roy Cardinal, Marie-Hélène, Porée, Jonathan, Soulez, Gilles, Ohayon, Jacques, Cloutier, Guy :A local angle compensation method based on kinematics constraints for non-invasive vascular axial strain computations on human carotid arteries.Computerized Medical Imaging and Graphics.38,123--136(2014)

16. Cherni, Mohamed Ali, Sayadi, Mounir, Fnaiech, Farhat: AI tools in medical image analysis: Efficacy of ANN for oestrogen receptor status assessment in immunohistochemical staining of breast cancer.International Journal of Biomedical Engineering and Technology.12,60--83(2013)

17. Wu, William K. K., Sung, Joseph J. Y.:Focus on gastrointestinal and liver cancers. J.SEMINARS IN CANCER BIOLOGY .23,469--470(2013) 
18. Berg, Bernd A.: Least square fitting with one explicit parameter less. J. COMPUTER PHYSICS COMMUNICATIONS.200,254--258(2016)

19. Prasad, Dilip K., Leung, Maylor K. H., Quek, Chai: ElliFit: An unconstrained, noniterative, least squares based geometric Ellipse Fitting method.J.PATTERN RECOGNITION.46,1449--1465(2013)

20. Shin, Youngsul, Choi, Yunja, Lee, Woo Jin: Integration testing through reusing representative unit test cases for high-confidence medicalsoftware. J.COMPUTERS IN BIOLOGY AND MEDICINE.43,434--443(2013) 